\title{
Sub-GHz Modulation of Light with Dielectric Nanomechanical Metamaterials
}

\author{
Artemios Karvounis $^{1 *}$, Jun-Yu Ou ${ }^{1}$, Behrad Gholipour ${ }^{1}$, Weiping Wu ${ }^{1}$, Kevin F. MacDonald ${ }^{1}$, \\ and Nikolay I. Zheludev ${ }^{1,2}$
}

\author{
${ }^{1}$ Optoelectronics Research Centre \& Centre for Photonic Metamaterials, University of Southampton, SO17 1BJ, UK \\ ${ }^{2}$ Centre for disruptive Photonic Technologies, Nanyang Technological University, Singapore \\ ak1c13@soton.ac.uk
}

\begin{abstract}
Subwavelength-thickness all-dielectric nano-grating and nano-cantilever array metamaterials, actuated respectively by electrostatic and optical forces, provide reversible reflectivity changes of up to $20 \%$ and a giant sub-GHz frequency optomechanical nonlinearity at telecommunication wavelengths.

OCIS codes: (160.3918) Metamaterials; (190.0190) Nonlinear optics; (120.4880) Optomechanics
\end{abstract}

We report on the first experimental demonstrations electrostatically (Fig. 1) and all-optically (Fig. 2) actuated, freestanding, all-dielectric nanomechanical metasurfaces. These subwavelength-thickness devices manufactured in CMOS-compatible media, provide in the former case reflectivity changes of up to $20 \%$ at applied biases of only a few volts, and in the latter a large optomechanical nonlinearity, operating at intensities of only a few $\mu \mathrm{W}$ per unit cell and a modulation frequencies of $152 \mathrm{MHz}$.

Non-metallic metamaterial nanostructures offer means of circumventing the losses and costs associated with the use of noble metals in traditional plasmonic architectures. As such they have attracted considerable attention of late, and it has been shown that oxides and nitrides, graphene, topological insulators, and high-index dielectrics can be used as platforms for the realization of high-Q resonant metamaterials. At the same time, it has been shown in plasmonic systems the reversible nanomechanical reconfiguration provides a powerful paradigm (harnessing the changing balance among Coulomb, Lorentz, Ampère, optical gradient and elastic forces at the nanoscale), for dynamically controlling the optical response of metasurfaces with high contrast.
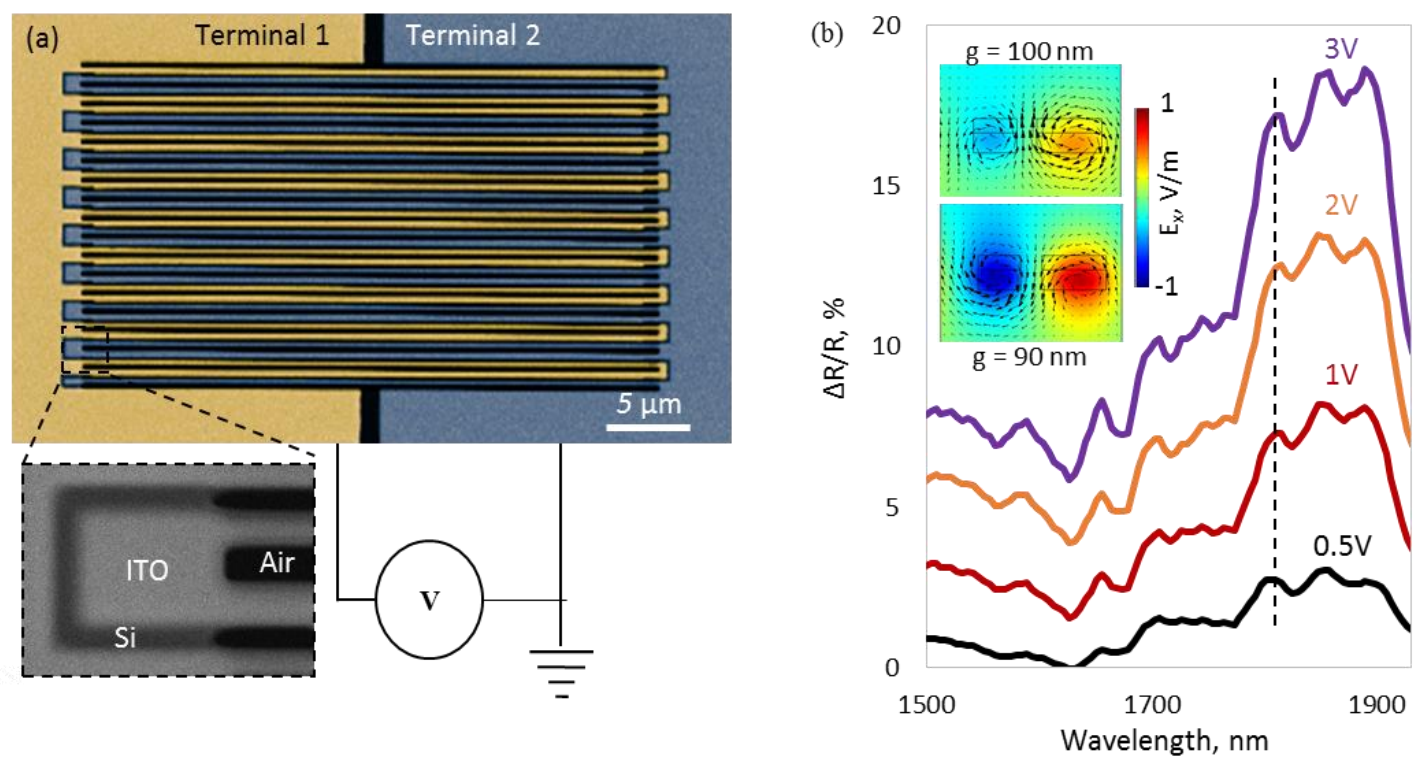

Fig. 1: Electrostatically controlled free-standing Si/ITO nano-grating metasurface for near-IR reflectivity switching: (a) False color scanning electron microscope image of a subwavelength grating metasurface in a silicon/ITO bilayer membrane . The different colors denote regions under opposing +/- electrical bias. (b) Experimentally measured dispersion relative reflection change for the nano-grating metasurface under a selection of applied bias levels [as labelled]. The insets show inset computationally simulated maps of electric field in the yz plane over a unit cell of the nano-grating [a pair of beams] at 1820 $\mathrm{nm}$ [dashed vertical line in the main panel] for beam separations $g$ of $100 \mathrm{~nm}$ [above] and $90 \mathrm{~nm}$ [below]. 
In this work we experimentally demonstrate all-dielectric metamaterials, fabricated in free-standing silicon and silicon/ITO bilayer nano-membranes, with resonant reflection and transmission characteristics that can be manipulated via the spatial reconfiguration of the constituent elements under the action of electrostatic and optical forces.

Electronically actuated subwavelength grating metasurfaces are manufactured, by focused ion beam milling, in a bilayer membrane of $100 \mathrm{~nm}$ thick polycrystalline silicon and $70 \mathrm{~nm}$ indium tin oxide (ITO). The ITO layer is structured such that an alternately positive/negative bias may be applied to alternate pairs of Si/ITO beams, as illustrated in Fig. 1a. The in-plane spacing of the beams (initially $100 \mathrm{~nm}$ ) is modified under the action of attractive/repulsive electrostatic forces between neighboring beams, substantially changing the optical properties of the metasurface array over a spectral band set by the dimensions of the beams: for light polarized parallel to the beams (exiting a resonance based on antiparallel displacement currents in the silicon component) the nano-grating structure of Fig. 1a presents a 20\% change in near-infrared reflectivity under an applied bias of 3V (Fig. 1b).

Optomechanically actuated metasurfaces are fabricated as an array of nano-cantilevers in a $100 \mathrm{~nm}$ thick freestanding polycrystalline silicon membrane (Fig. 2a). The structure is designed such that illumination at a pump wavelength of $1550 \mathrm{~nm}$ generates optical forces (via the excitation of a predominantly electric dipolar resonance) that tilt the cantilever arms out-of-plane, bringing about a change in the transmission of the array at a probe wavelength of $1310 \mathrm{~nm}$ (by means of spectrally shifting the magnetic resonance excited in that waveband).

The static displacement of the cantilevers is negligibly small but they are driven to oscillate at much higher amplitude when the pump beam is modulated at a mechanical eigenfrequency of the structure - the response being enhanced by the structure's mechanical quality factor of $\sim 100$. In consequence the metasurface provides an extremely large optomechanical nonlinearity (an effective susceptibility $\operatorname{Im}\left\{\chi^{(3)}\right\} / n^{2} \sim 3.9 \times 10^{-14} \mathrm{~m}^{2} \mathrm{~V}^{-2}$ ) at intensities of only a few tens of $\mu \mathrm{W} / \mu \mathrm{m}^{2}$ and at modulation frequency of $152 \mathrm{MHz}$.

(a)

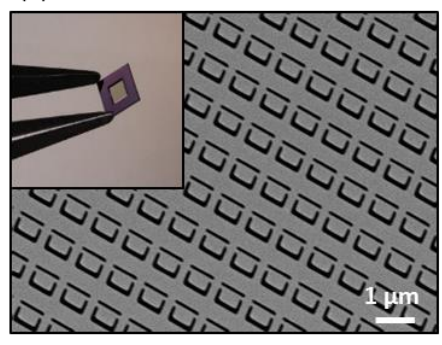

(b)

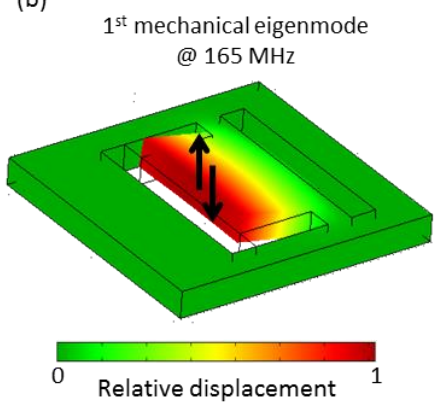

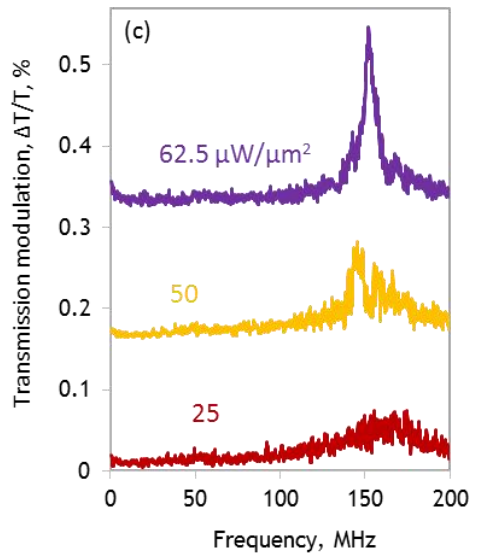

Fig. 2: Giant optomechanical nonlinearity in a free-standing silicon nano-cantilever array driven by optical forces: (a) Scanning electron microscope image of a free-standing silicon membrane nano-cantilever metasurface array [dark areas being slots cut through the membrane]. The inset photograph shows the $100 \mathrm{~nm}$ thick membrane window in it s $10 \mathrm{~mm} \times 10 \mathrm{~mm}$ bulk silicon frame. (b) Numerically simulated nano-cantilever unit cell colored according to the relative magnitude of out-of-plane displacement during oscillation at the structure's first mechanical eigenfrequency (c) $1310 \mathrm{~nm}$ probe transmission modulation depth as a function of $1550 \mathrm{~nm}$ pump modulation frequency for a selection of peak pump intensities [as labelled].

Ultrathin all-dielectric nanomechanically reconfigurable photonic metasurfaces exploit the unique technological and manufacturing opportunities provided by dielectric/semiconductor membrane platforms and offer a compact, energy efficient and fast active optoelectronic platform potentially suited to practical application in high speed photonic applications. 